\title{
Entrenamiento integrado aplicado en cancha - Estudios de casos ¿Es la metodología del futuro?
}

\author{
Piotr Unierzyski, Mieczysław Bogusławski y Simon Wheatley
}

\section{RESUMEN}

Este documento discute el coaching integrado, un enfoque que se deriva de la teoría de que el desempeño experto se basa en los diferentes aspectos del juego (físico, psicológico, técnico y táctico) utilizados instintivamente y en conjunto por el atleta, y que por lo tanto la mejor manera de crear expertos habilidades y rendimiento es entrenar estos aspectos juntos. Se discuten ejemplos prácticos y casos de estudio.

\author{
Palabras clave: entrenamiento \\ integrado, desempeño \\ experto, desarrollo de \\ habilidades \\ Recibido: 19 Mayo 2018 \\ Aceptado: 25 Junio 2018 \\ Autor correspondiente: \\ Piotr Unierzyski. \\ Correo electrónico: \\ piotrunierzyski@wp.pl
}

\section{INTRODUCCIÓN}

La diferencia entre los mejores jugadores de tenis y todos los otros se comenta a diario entre los entrenadores, los jugadores y los padres. Los resultados de ciertos estudios (Schönborn 1984, 1994, Unierzyski 2011) definen la coordinación-agilidadvelocidad, la potencia, las habilidades tácticas, técnicas y mentales como los factores más importantes (limitantes) que inciden sobre el rendimiento tenístico. La investigación demuestra que los mejores jugadores poseen un alto nivel de todos estos factores (y sin grandes brechas), ya desde la etapa de juniors.

Respetando estos resultados, preferimos mirar el éxito en el tenis desde una perspectiva algo diferente y sacar las conclusiones para la práctica. Profundizando un poquito, y desde el punto de vista del tenis (utilizando la filosofía basado en el juego), el rendimiento de los mejores jugadores se puede describir así:

- Toman un alto porcentaje de decisiones

- Golpean la pelota con "suficiente" potencia y con un alto grado de precisión.

- Tienen un estilo de juego claramente definido con patrones y combinaciones de tiros específicos y preferidos
- Tienen "armas" y súper fortalezas

- Tienen grandes habilidades para la anticipación

- Además, pueden improvisar cuando es necesario, tomar decisiones no ortodoxas, y ejecutar tiros igualmente no ortodoxos. En esencia, tienen grandes habilidades de adaptación.

- Pueden jugar bien las 3 fases de juego, sabiendo cuándo y cómo atacar mejor, pelotear, defender y contra atacar.

Desarrollando la teoría de Pierre Bourdieu y el enfoque del entrenamiento basado en el juego/ táctico, podemos explicar que los grandes campeones pueden conectar instintivamente decisiones tácticas, técnicas fluidas y tiros precisos en un proceso efectivo, de tal modo que las reacciones se tornan automáticas (Bourdieu, 1990; Crespo y Cooke, 1999). Pueden utilizar las habilidades específicas para el juego (técnicas, físicas, de coordinación y mentales), todas juntas, al mismo tiempo, de manera que se sustenten entre sí. Esta destreza, una combinación de habilidades instintivas y complejas se podría llamar "super competencia" o, "habitus" según Bourdieu.

Muchas afirmaciones sugieren que la separación entre el entrenamiento mental, físico y táctico- técnico en el nivel de 
alto rendimiento es "más artificial que natural" (Crespo y Reid, 2002). Es lógico que si todas estas habilidades diferentes deben trabajar juntas, los jugadores y los entrenadores deberían trabajarlas de la manera más integrada posible.

La pregunta es: ¿cómo hacerlo, cómo enseñarlas y cómo hacer que nuestros jugadores aprendan y se formen de la mejor manera?

Algunos entrenadores continúan trabajando del modo tradicional, utilizando métodos que no son específicos para el juego, y por lo tanto, separados.

Los ejemplos clásicos son:

- El entrenamiento físico

- Utilizando corridas de larga distancia- para crear una base aeróbica, como recomiendan los mejores métodos para todos los niveles de jugadores.

- Trabajando la "flexibilidad general" o la fuerza en un gimnasio solamente

- La preparación mental - trabajando con los jugadores en sus oficinas- lejos de la situación real de partido

- Entrenamiento en cancha- utilizando ejercicios cerrados como la herramienta principal para desarrollar las habilidades tácticas y técnicas.

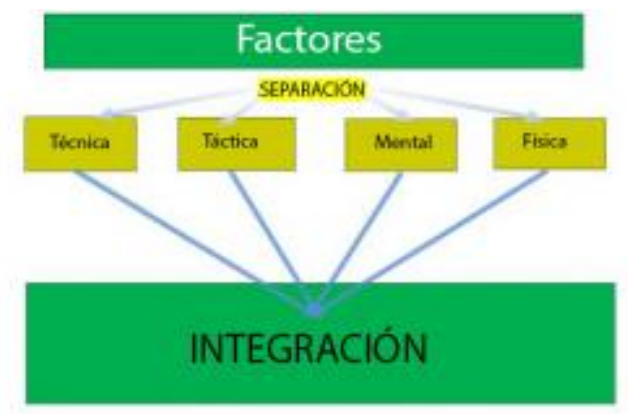

Figura 1. Diferentes aspectos del entrenamiento de tenis e integración para un enfoque holística.

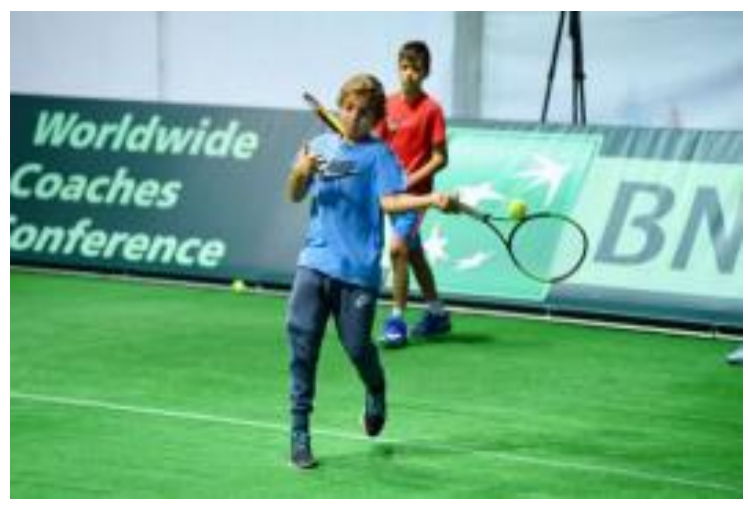

Utilizar métodos tomados del atletismo, o del entrenamiento personal, sin respetar el "modelo" de juego, lleva a mejorar la aptitud física general, pero usualmente no ayuda demasiado a mejorar el rendimiento en cancha. El mismo o mejor resultado se puede lograr por medio de la práctica específica en cancha, y cuando los expertos/entrenadores de diferentes áreas (física, mental, técnica y táctica) trabajan en cancha en conjunto.

Recientemente, hemos podido observar que los métodos de enseñanza tradicional y conservadores se están enriqueciendo con otros más nuevos. Los esfuerzos de los entrenadores líderes adaptan los métodos de entrenamiento a las exigencias específicas del juego más que antes, el concepto de entrenamiento integrado se acepta cada vez más.

El objetivo final de tal enfoque de entrenamiento es enseñar a los jugadores a conectar las decisiones tácticas, las técnicas fluidas y la precisión de los tiros de manera instintiva. Para lograrlo, los entrenadores modernos utilizan ejercicios complejos para consolidar e integrar la preparación mental con las habilidades técnicas y tácticas. El entrenamiento integrado permite que el entrenador con conocimiento elija mejor las combinaciones de ejercicios diseñados individualmente, según las necesidades de los jugadores, manteniendo el régimen de energía específico para el juego.

Todo el proceso comienza a partir de un análisis profundo del rendimiento de los jugadores en situaciones abiertas. Las estadísticas de partidos podrían ser un buen punto de inicio pero, idealmente, la razón para el mejor o peor rendimiento debe investigarse más profundamente. Por ejemplo, las razones para el mejor o peor rendimiento pueden ser:

- Mentales (ej. actitud, falta de focalización, control emocional, falta de motivación)

- Físicas (estabilidad del torso, potencia, fuerza, velocidad)

- Tácticas (cómo jugar de manera efectiva contra un zurdo, patrones, toma de decisiones, etc.)

- Técnicas

- habilidades de recepción (trabajo con la raqueta, trabajo corporal, trabajo de pies)

- habilidades de envío (trabajo con la raqueta, trabajo corporal, trabajo de pies).

Entonces, comenzamos por un aspecto mental, por ejemplo, la actitud, luego la táctica, (¿cuál es tu plan?), seguido del análisis físico y técnico. La Observación/ Análisis y la intervención posible deben tener en cuenta los 4 factores para el rendimiento. Es importante entrenar en colaboración con el deportista, utilizando por ejemplo, preguntas para evaluar al jugador en una situación de juego específica. Luego, se deben definir los objetivos y el plan de entrenamiento para el jugador. 


\section{ESTUDIOS DE CASOS}

\section{Ejemplo 1}

Esto se puede ilustrar mejor con un ejemplo de la vida real, de un jugador que haya elevado el nivel de su juego enormemente, en solamente un año.

- Una jugadora elite (WTA) clasificada 400 con la aspiración de estar entre las 100 mejores

- Relativamente pequeña (sin grandes armas)

- Gran actitud, ética de trabajo

- Para ser más eficiente tiene que ser más agresiva, tener más juego de fondo, para que sus adversarias corran más y comentan más errores.

- Mentalmente, y físicamente, tiene que correr más velozmente, y estar dispuesta a jugar largos peloteos y partidos.

- Debe poder jugar peloteos con 8-10 tiros o más (o sea, sobre el promedio para el tenis femenino)

- Además, tiene que poder jugar 4-5 partidos en una semana, al mismo nivel, o sea, tiene que poder recuperar rápidamente.

Entonces, su velocidad y su resistencia específica para el tenis (que se contrastan entre sí) deben ser mejores que las de sus adversarias, y los movimientos más económicos para superar y resistir más en los peloteos que sus adversarias, y finalmente, poder jugar todo el torneo, no solamente uno o dos partidos.

De acuerdo con los métodos de entrenamiento tradicionales, podría correr mucho fuera de la cancha para desarrollar una base aeróbica, y usar el gimnasio para fortalecer su cuerpo y trabajar en sus habilidades tácticas y técnicas separadamente, aisladas de la fuerza y del acondicionamiento.

En cambio, ella ha enriquecido su programa e incluido una o dos series de entrenamiento integrado por semana, dentro de su práctica regular en cancha.

Por ejemplo:

- Series de 6-8 bloques, cada uno consta de un ejercicio de fuerza y acondicionamiento seguido por un ejercicio de tenis (cerrado o más abierto según el objetivo del entrenamiento):

- Práctica dirigida a desarrollar la velocidad y la potencia específicamente para el "tenis".
- 6-10 segundos de ejercicios de fuerza y acondicionamiento seguidos por un ejercicio de tenis de 6 a 10 tiros.

- Práctica dirigida al desarrollo de la resistencia/ vigor específicos

- 10 -20 segundos de ejercicios seguidos por un ejercicio de tenis de 12 a 18 tiros.

A cada bloque le seguirá un descanso hasta la recuperación de la fosfocreatina.

A tal práctica le seguirá un partido de práctica.

Toda la práctica deberá ser desafiante, pero no agotadora, (ácido láctico entre 6-8 mili moles)

- Permite dar forma a todos los parámetros (factores que limitan el rendimiento) en muy poco tiempo.

- Acelera el proceso de entrenamiento

- Ahorra tiempo

Es clave enseñar la técnica de movimientos adecuada antes de trabajar la velocidad y la dinámica.

Resultados de cada práctica:

Absorción máxima de oxígeno (Vo2 max) elevada a 59 $\mathrm{ml} / \mathrm{kg} / \mathrm{min}$ - también se eleva la velocidad de la acción y de la corrida.

(Sin ningún entrenamiento convencional de larga distancia o intervalos).

La jugadora confesó:

"No podía ganar más de dos partidos seguidos."

"No sé qué me sucedió pero... estoy mucho más rápida, en mejor forma física, más dinámica y juego mucho mejor.

"He ganado 13 partidos, muchos en 3 sets, solamente con dos meses de esa práctica."

"Ascendí del 600 al 180 de la WTA en muy poco tiempo."

"¡El peloteo más largo que gané duró más de 45 tiros! - mi adversaria "murió" pero yo pude jugar normalmente después de eso."

"No significa que nunca tenga que ir al gimnasio, pero no necesita la carrera de larga distancia para mejorar su resistencia."

Ejemplo 2

Un jugador elite de 11 años (entre los mejores 3 de Polonia) 
Metas de entrenamiento:

Desarrollar un estilo de juego ofensivo, completo, hacer que el adversario corra utilizando ambos golpes de fondo, y mejorar el control emocional por medio de rutinas de aprendizaje en cancha.

Por ejemplo:

- Series de 6 bloques, cada uno consta de uno específico para "tenis" velocidad/agilidad y dura 4-6 segundos, seguido por un ejercicio para tenis cerrado (series de 4 - 5 $-6-5$ - 4 tiros, cruzados a objetivos, realizados con intensidad moderada)

- Esto será seguido de un descanso hasta la recuperación total (frecuencia de pulso debajo de 90 latidos por minuto)

- El jugador debe mostrar una conducta de rutina tras cada ejercicio de tenis.

- Le siguen 20 minutos de partido de práctica (ej. super muerte súbita)

\section{CONCLUSIÓN}

Una de las ventajas de estas prácticas (en ambos ejemplos) es que el cuerpo, cuando trabaja bajo condiciones desafiantes, comienza a funcionar de una manera más económica. La observación muestra que algunos músculos innecesarios se "apagan" y solo los más importantes, responsables de realizar una técnica determinada, funcionan con mayor fluidez. Como resultado, la técnica e incluso la precisión del disparo aumentan junto con la velocidad / potencia y la resistencia específicas.

El cambio de comportamiento resultante también es relativamente duradero, y también es suficiente para realizar este tipo de sesión una vez por semana dentro de un programa de entrenamiento normal pero estructurado con el fin de obtener adaptaciones positivas.

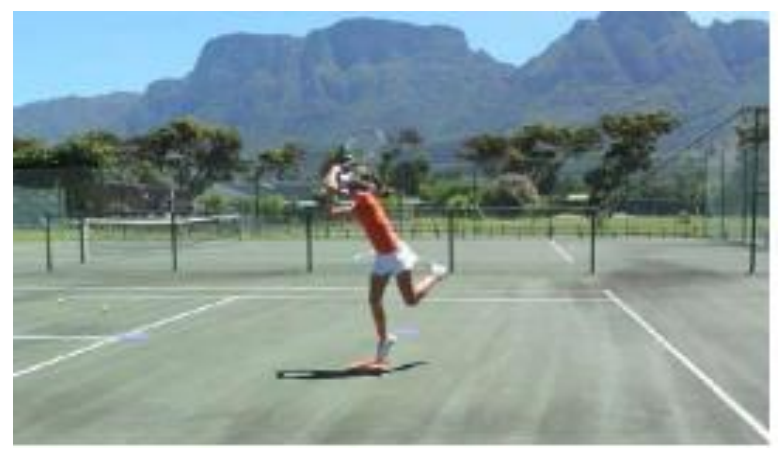

La práctica o entrenamiento integrado debe verse como una forma más efectiva de transferir habilidades más generales (mentales, físicas y de coordinación) a las habilidades específicas del tenis. También permite una mayor consolidación e integración de todos los parámetros / factores de rendimiento (factores que limitan el rendimiento) en un período de tiempo más corto y de una manera más completa, lo que podría significar que creará un jugador más completo. Acelera el proceso de capacitación, lo que significa que los jugadores ven los resultados más rápido y, como resultado, pueden estar más felices con su progreso y también pueden dedicar más tiempo a otras actividades esenciales, incluida la prevención de lesiones. Este tipo de entrenamiento también puede ser el siguiente paso en el enfoque centrado en el jugador, ya que implica el entrenamiento de una manera más holística, poniendo a la "persona" primero, antes que al "jugador".

\section{REFERENCIAS}

Bourdieu, P. (1990).The Logic of Practice. Cambridge Polity Press.

Cayer, L. (2017) Personal communication.

Crespo, M., and Cooke K. (1999). "The Tactical Approach to Coaching Tennis". ITF Coaches Review, Issue 19, 7-8.

Crespo, M., and Reid M. (2002). "Modern tactics: an introduction". ITF Coaching \& Sport Science Review. 27, 2.

Kovacs, M. S. (2006) . Applied physiology of tennis performance $(\mathrm{Br} J$ Sports Med 2006;40:381-386. https://doi.org/10.1136/bjsm.2005.023309

Schönborn, R. (1984). Leistungslimitierende und Leistungsbestimmende Faktoren. (In:) H. Gabler, B. Zein (eds), Talentsuche und Talent Förderung im Tennis. Beitraege vom 1. Symposium des Sportwissenschaftlichen Beirats des DTB 1983, Ahrensburg: Czwalina, 51-75.

Schönborn, R. (1993). Players' performance and development. ITF Coaches Review, 2, 1-2.

Schönborn, R. (1994). Modern Complex Training in Tennis. ITF Coaches Review, 4, 2-3.

Unierzyski, P. (1994). Relations Between Experience, Fitness, Morphological Factors and Performance Level with Reference to the Age. ITF Sport Science and Coaches Review, no. 3, 8.

Unierzyski, P. (2011) Retrospective analysis of factors affecting performance in tennis. (in Polish). Monografia 397. AWF, Pozna 
CONTENIDO ITF ACADEMY RECOMENDADO (HAZ CLICK ABAJO)

\section{TTF Academy}

Derechos de Autor (c) 2018 Piotr Unierzyski, Mieczysław Bogusławski y Simon

\author{
Wheatley
}

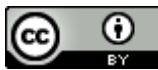

Este texto está protegido por una licencia CreativeCommons $\underline{4.0 .}$

Usted es libre para Compartir - copiar y redistribuir el material en cualquier medio o formato-y Adaptar el documento — remezclar, transformar y crear a partir del material- para cualquier propósito, , incluso para fines comerciales, siempre que cumpla la condición de:

Atribución: Usted debe dar crédito a la obra original de manera adecuada, proporcionar un enlace a la licencia, e indicar si se han realizado cambios. Puede hacerlo en cualquier forma razonable, pero no de forma tal que sugiera que tiene el apoyo del licenciante o lo recibe por el uso que hace de la obra.

Resumendelicencia - Textocompletodelalicencia 